\title{
Mittag-Leffler String Stability of Singularly Perturbed Stochastic Systems within Local Fractal Space
}

\author{
Khosro Sayevand \\ Faculty of Mathematical Sciences, Malayer University \\ P. O. Box 16846-13114, Malayer, Iran \\ E-mail(corresp.): ksayehvand@malayeru.ac.ir
}

Received June 6, 2018; revised March 8, 2019; accepted March 9, 2019

\begin{abstract}
The present paper tends to define a new type of string stability based on Mittag-Leffler function that is called $(p \alpha)$-string stability. This kind of stability will be considered for a class of singularly perturbed stochastic systems of fractional order. The fractional derivative in these systems is situated in the local sense. String stability indicates uniform boundedness of the interconnected system, if the initial cases of interconnected system be uniformly bounded. The deduction of the sufficient conditions of stability is based on a mixture of the concept of the Mittag-Leffler stability with the notion of $p$-mean string stability of singularly perturbed stochastic systems. In this sense the objective, it is argued, is to investigate the full order system in their lower order subsystems, i.e., the reduced order system and the boundary layer correction.
\end{abstract}

Keywords: Mittag-Leffler stability, string stability, singular perturbation, stochastic systems, local fractional derivative.

AMS Subject Classification: 34A08; 93E15; 34D15.

\section{Introduction}

The fractional calculus is a generalization of the classical calculus and the history of it traces back to more than 300 years ago. From then on, several mathematicians contributed to the expansion of it, including Riemann, Liouville, Abel, Grünwald, Letnikov, Weyle and Riesz. Today, fractional calculus is a subject undergoing intense study with rapid progress and executions in different areas of science and engineering. For more details see [2] and the references therein. 
The topic of stability analysis of nonlinear singularly perturbed stochastic systems was recently investigated in [26]. The sufficient conditions of uniform, stochastic asymptotic stability for a special class of nonlinear, singularly perturbed systems were obtained in $[6,7]$. However, stability analysis of the mentioned systems has not been widely developed in the scope of fractional calculus. This paper attempts to conduct a detailed study of sufficient conditions so as to present a new type of Mittag-Leffler stability for nonlinear, singularly perturbed stochastic systems of fractional order. In order to match this type of stability to the scope of fractional differential systems, we employ a relatively new derivative that is called the local fractional derivative.

We use the concept of $p$-stability $[12,21]$ and blend it with the idea of the global uniform Mittag-Leffler stability $[1,3,15]$ to deduce new results called Mittag-Leffler string stability for the mentioned systems. The main result is obtained in the form of Definition 2. The subsequent results of this definition are given and we indicate that when sufficient conditions of Mittag-Leffler string stability are fulfilled for both the reduced order system and the boundary layer system and also some further assumptions, the full order system is MittagLeffler string stable for sufficient small values of perturbation parameter.

Our motivation to investigate the stochastic systems within local fractal space [34] is that, in a new study, scientists have found that a serial killer's pattern of murders seems to conform to a strict mathematical formula [24]. They theorize that the reason murders are associated with a mathematical function known as 'the Devil's staircase' is that serial killers patterns of behavior driven by firing of neurons in their brains. This research, by using data from a Russian serial killer Andrei Chikatilo, nicknamed Rostov Ripper, whose 53 murders are well known, seems to offer the possibility that police could be ready when other killers were driven to strike. Moreover, murder patterns of other serial killers are similar to that of Chikatilo, cf. Figures 5 and 6 of [24] which show the cumulative number of murders committed by Yang Xinhai, and Moses Sithole. However, the researchers acknowledge that prediction measurements need to be refined. We must points out that, the Devil's staircase is a statistical term referring to graph results that resembles a staircase of increasing values when plotted out. The plot of the cumulative number of murders committed by Chikatilo over 12 years, which is a Devil's staircase [24] is shown here by Figure 1. Mathematically, the results depict a function that is partially continuous. The analysis of differentiability is usually given by means of fractal dimension, via the Hausdorff dimension that appears to be the most popular choice. The Hausdorff dimension of the set of non-differentiability of the Devil's staircase is $\ln 2 / \ln 3$.

\section{A brief review of prerequisites based on local fractional calculus and probability theory}

Recently, the fractional calculus is sufficiently used in probability theory and stochastic systems. For instance, the probability density of fractional order 


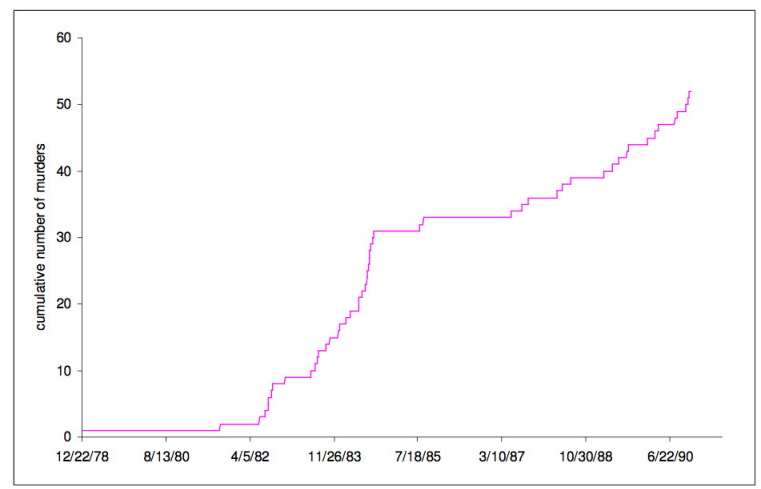

Figure 1. The plot of the cumulative number of murders committed by Chikatilo over 12 years, which is a Devil's staircase [24].

$f_{\alpha}(x)$ namely that

$$
F[a, b]:=P[a<X<b]=\int_{a}^{b} f_{\alpha}(t)(d t)^{\alpha}, 0<\alpha<1,
$$

is investigated by means of fractional calculus [8]. Furthermore, by using the uniform fractional probability density function, the classical probability axioms are validated for a fractional probability measure. For more details, the reader is recommended to consult the research works presented in [19,27]

The idea of local fractional calculus $[22,32,33]$, which was first proposed by Kolwankar and Gangal [14] based on the Riemann-Liouville fractional derivative [13], was employed to deal with non-differentiable problems in science and engineering [29]. Yang et al. [28, 29,30,31] presented the logical generalizations of the definitions to the subject of local derivative on fractals. First, we recall the basic notations and definitions.

A function $f(x)$ is called local fractional continuous at $x=x_{0}$, if for each $\epsilon>0$ there exists $\delta>0$ such that, $[28,29,30,31]$,

$$
\left|f(x)-f\left(x_{0}\right)\right|<\epsilon^{\alpha}, \quad 0<\alpha \leq 1,
$$

whenever $\left|x-x_{0}\right|<\delta$. It is written as $\lim _{x \rightarrow x_{0}} f(x)=f\left(x_{0}\right)$.

$f(x)$ is called local fractional continuous on the interval $(a, b)$, denoted by $f(x) \in C_{\alpha}(a, b)$, if $(2.1)$ is valid for $x \in(a, b)$.

Let $f(x) \in C_{\alpha}(a, b)$. Local fractional derivative of $f(x)$ of order $\alpha$ at $x=x_{0}$ is defined as, $[28,29,30,31]$,

$$
f^{(\alpha)}\left(x_{0}\right)=\left.\frac{d^{\alpha} f(x)}{d x^{\alpha}}\right|_{x=x_{0}}=\lim _{x \rightarrow x_{0}} \frac{\Delta^{\alpha}\left(f(x)-f\left(x_{0}\right)\right)}{\left(x-x_{0}\right)^{\alpha}}, \quad 0<\alpha \leq 1,
$$

where $\Delta^{\alpha}\left(f(x)-f\left(x_{0}\right)\right) \cong \Gamma(\alpha+1)\left(f(x)-f\left(x_{0}\right)\right)$. Here, $\Gamma(\cdot)$ is a gamma function. 
Suppose that for any point $x \in(a, b)$ there exists

$$
f^{(\alpha)}(x)=\frac{d^{\alpha} f(x)}{d x^{\alpha}}=D_{x}^{\alpha} f(x) .
$$

In this case, $D_{x}^{\alpha}(a, b)$ is called a $\alpha$-local fractional derivative set and

$$
f(x) \in D_{x}^{\alpha}(a, b)
$$

Local fractional derivative meets the following simple rules

$$
D_{x}^{\alpha} c=0, \quad D_{x}^{\alpha}[c f]=c D_{x}^{\alpha} f, \quad D_{x}^{\alpha} x^{\beta}=\frac{\Gamma(1+\beta)}{\Gamma(1+\beta-\alpha)} x^{\beta-\alpha}, \quad \beta \geq \alpha>0,
$$

and the following simple chain rules [28, 29,30,31],

$$
D_{x}^{k \alpha} f(x)=\overbrace{D_{x}^{\alpha} D_{x}^{\alpha} \ldots D_{x}^{\alpha}}^{k \text { times }} f(x), \quad D_{x}^{\alpha}[(f \circ g)(x)]=\left(\frac{d g}{d x}\right)^{\alpha} D_{x}^{\alpha} f(g(x)) .
$$

Let $f(x) \in C_{\alpha}(a, b), \quad 0<\alpha \leq 1$. Local fractional integral of $f(x)$ of order $\alpha$ in the interval $[a, b]$ is given by $[28,29,30,31]$,

$$
{ }_{a} I_{b}^{(\alpha)} f(x)=\frac{1}{\Gamma(\alpha+1)} \int_{a}^{b} f(x)(d x)^{\alpha}=\frac{1}{\Gamma(\alpha+1)} \lim _{\Delta x \rightarrow 0} \sum_{j=0}^{N-1} f\left(x_{j}\right)\left(\Delta x_{j}\right)^{\alpha},
$$

where $\Delta x_{j}=x_{j+1}-x_{j}, \Delta x=\max \left\{\Delta x_{1}, \Delta x_{2}, \ldots, \Delta x_{j}\right\}$, and $\left[x_{j}, x_{j+1}\right], j=$ $0,1, \ldots, N-1, x_{0}=a, x_{N}=b$, is a partition of the interval $[a, b]$.

Suppose that for any point $x \in(a, b)$ there exists ${ }_{a} I_{x}^{\alpha} f(x)$. In this case, $I_{x}^{\alpha}(a, b)$ is called a $\alpha$-local fractional integral set and

$$
f(x) \in I_{x}^{\alpha}(a, b) .
$$

Let $F$ be a subset of the real line and be a fractal (e.g., Cantor set or likeCantor set). Following (2.2), the $\alpha$-dimensional Hausdorff measure $H^{\alpha}$ is given by $[28,29,30,31]$,

$$
H^{\alpha}\left(F \cap\left(x_{0}, x\right)\right)=\left(x-x_{0}\right)^{\alpha}, \quad 0<\alpha \leq 1,
$$

and its plot when $\alpha=\frac{\ln 2}{\ln 3}$ is the dimension of the fractal set $F$ and $x_{0}=0$.

The Mittag-Leffler function which is named after the Swedish mathematician Gösta Mittag-Leffler, who defined it in 1903 [18], plays a major role in the rest of this paper. It should be noted that, in fractional calculus, the MittagLeffler function, as a similar to exponential naturally function in classical calculus, plays an essential task $[10,11]$. Carries out, the exponential function itself is a particular case of the Mittag-Leffler function. We recall that the twoparametric Mittag-Leffler function in fractal space is defined as $[28,29,30,31]$,

$$
E_{\alpha, \beta}\left(x^{\alpha}\right)=\sum_{n=0}^{\infty} \frac{x^{n \alpha}}{\Gamma(n \alpha+\beta)}, \quad x \in \mathbb{R}, \quad 0<\alpha \leq 1, \quad \beta \in \mathbb{R},
$$


and for $\beta=1$, the above definition reduces to the Mittag-Leffler function in one parameter

$$
E_{\alpha, 1}\left(x^{\alpha}\right)=\sum_{n=0}^{\infty} \frac{x^{n \alpha}}{\Gamma(n \alpha+1)} \equiv E_{\alpha}\left(x^{\alpha}\right), \quad x \in \mathbb{R}, \quad 0<\alpha \leq 1 .
$$

It is worth mentioning that, for $0<\alpha \leq 1$ the following semigroup properties via Mittag-Leffler function are defined on the fractal set $F$ hold $[28,29,30$, 31]:

$$
\begin{aligned}
& E_{\alpha}\left(x^{\alpha}\right) E_{\alpha}\left(y^{\alpha}\right)=E_{\alpha}\left(x^{\alpha}+y^{\alpha}\right)=E_{\alpha}\left((x+y)^{\alpha}\right), \quad x, y \in \mathbb{R}, \\
& E_{\alpha}\left(x^{\alpha}\right) E_{\alpha}\left(-y^{\alpha}\right)=E_{\alpha}\left(x^{\alpha}-y^{\alpha}\right)=E_{\alpha}\left((x-y)^{\alpha}\right), \quad x, y \in \mathbb{R} .
\end{aligned}
$$

Analogous to exponential stability, the Mittag-Leffler stability is a popular property used to show stability and boundedness of solutions of fractional differential equations. Now, let us consider the following equation

$$
\frac{d^{\alpha} x}{d t^{\alpha}}=F(x, t), \quad 0<\alpha \leq 1
$$

with the initial condition

$$
x\left(t_{0}\right)=x_{0}, \quad t_{0} \leq t<\infty,
$$

where $F$ is an arbitrary function. The solution of equation (2.4)-(2.5) is said to be Mittag-Leffler stable $[1,15]$, if

$$
\|x(t)\| \leq\left\{m\left[x\left(t_{0}\right)\right] E_{\alpha}\left(-\lambda\left(t-t_{0}\right)^{\alpha}\right)\right\}^{\beta},
$$

where $\lambda \geq 0, \beta>0, m(0)=0, m(x) \geq 0$. In addition, $m(x)$ is locally Lipschitz on $x \in B \subset \mathbb{R}^{n}$ with the Lipschitz constant $m_{0}$.

Lemma 1. Let $x(t)$ be a local fractional continuous for $t \geq 0$, such that the following condition is satisfied for almost all $t \geq 0$,

$$
\frac{d^{\alpha} x}{d t^{\alpha}} \leq A(t) x+B(t), \quad 0<\alpha \leq 1
$$

where we assumed that $A(t), B(t)$ are almost everywhere fractional continuous and fractional integrable over all finite intervals. Then

$$
x(t) \leq x(0) E_{\alpha}\left(\int_{0}^{t} A(s)(d s)^{\alpha}\right)+\int_{0}^{t} E_{\alpha, \alpha}\left(\int_{s}^{t} A(u)(d u)^{\alpha}\right) B(s)(d s)^{\alpha} .
$$

Proof. It is similar to the proof of Example (4.9; page 231) of [13].

Lemma 2. Consider the symmetric matrix $M(\epsilon)=\left[m_{i j}(\epsilon)\right], i, j=1,2$, where the function $m_{i j}:(0, \infty) \rightarrow \mathbb{R}$ fulfils

$$
\lim _{\epsilon \rightarrow 0} m_{11}(\epsilon)=\lambda_{0}, \quad \lim _{\epsilon \rightarrow 0} m_{22}(\epsilon)=\infty, \quad \lim _{\epsilon \rightarrow 0} \frac{m_{12}^{2}(\epsilon)}{m_{22}(\epsilon)}=0 .
$$

Then $\lim _{\epsilon \rightarrow 0} \beta_{\min }(M(\epsilon))=\lambda_{0}$, where $\beta_{\min }(M(\epsilon))$ is the minimal eigenvalue of matrix $M(\epsilon)$. 
Proof. See [25].

Let $W$ be a probability space, i.e. a triple $W=(\Omega, \mathfrak{A}, \mathbf{P})$, where $\Omega$ is the sample space, $\mathfrak{A}$ is its $\sigma$-algebra and $\mathbf{P}$ is a probability measure. For more details, see $[4,5,12,17]$.

A random variable is a $\mathfrak{A}$-measurable and almost everywhere finite function $\xi(\omega)$ on $\Omega$. In this paper we will consider only random variables which obtain values in Euclidean $l$-space $\mathbb{R}^{l}$, namely, such that $\xi(\omega)=\left(\xi_{1}(\omega), \ldots, \xi_{l}(\omega)\right)$ be a vector in $\mathbb{R}^{l}(l=1,2, \ldots)$.

The expectation of a random variable $\xi(\omega)$ is defined as follows

$$
\mathbf{E} \xi=\int_{\Omega} \xi(\omega) \mathbf{P}(d \omega)
$$

provided the function $|\xi(\omega)|$ is integrable.

Assume that $\mathfrak{B}$ be a $\sigma$-algebra of Borel subsets of a closed interval $I=$ $\left[s_{0}, s_{1}\right]$, and $\mathfrak{B} \times \mathfrak{A}$ be the minimal $\sigma$-algebra of subsets of $I \times \Omega$ including all subsets of the kind $\{t \in \Delta, \omega \in A\}$, where $\Delta \in \mathfrak{B}, A \in \mathfrak{A}$. A function $\xi(t, \omega) \in \mathbb{R}^{l}$ is known as a measurable stochastic process or random function, defined on $\left[s_{0}, s_{1}\right]$ with values in $\mathbb{R}^{l}$ if it is $\mathfrak{B} \times \mathfrak{A}$-measurable and $\xi(t, \omega)$ be a random variable for each $t \in\left[s_{0}, s_{1}\right]$. For fixed $\omega$, we will call the function $\xi(t, \omega)$ a trajectory or sample function of the stochastic process. As a result, we will consider only separable stochastic processes, namely, the processes whose behavior for all $t \in\left[s_{0}, s_{1}\right]$ is determined probability zero happens by its behavior on some dense subset $\Lambda \in\left[s_{0}, s_{1}\right]$. For more details, see $[4,5,12,17]$.

$\xi(t, \omega)$ is called stochastically continuous at $s \in\left[s_{0}, s_{1}\right]$, if for each $\epsilon>0$

$$
\lim _{t \rightarrow s} \mathbf{P}\{|\xi(t, \omega)-\xi(s, \omega)|>\epsilon\}=0 .
$$

For any process $\xi(t, \omega)$ which is stochastically continuous all over $\left[s_{0}, s_{1}\right]$, except for a countable subset of $\left[s_{0}, s_{1}\right]$, there is a separable measurable process $\tilde{\xi}(t, \omega)$, which for all $t \in\left[s_{0}, s_{1}\right]$ ( [4] Chap. 2)

$$
\mathbf{P}\{\xi(t, \omega)=\tilde{\xi}(t, \omega)\}=1, \quad(\xi(t, \omega)=\tilde{\xi}(t, \omega) \text { almost surely }) .
$$

If the stochastic process $\xi(t, \omega)$ be measurable, then $\xi(t, \omega)$ is almost surely Lebesgue-measurable for fixed $\omega$. Also, if $\mathbf{E} \xi(t, \omega)$ exists, then it is Lebesguemeasurable, i.e.,

$$
\int_{A} \mathbf{E}|\xi(t, \omega)| d t<\infty
$$

and $\xi(t, \omega)$ is almost surely integrable on $A$. See Chap. 2 of [4].

Theorem 1. The trivial solution of the following system

$$
\frac{d^{\alpha} x}{d t^{\alpha}}=f(x, t)+q(x, t) \xi(t), 0<\alpha \leq 1, x \in \mathbb{R}^{n}
$$

is Mittag-Leffler $(p \alpha)$-stable for $t \geq 0$ if there is a function $V(x, t)$ of class $\mathbf{C}_{\alpha}^{0}(E)$ such that

$$
\begin{aligned}
& \kappa_{1}|x|^{p \alpha} \leq V(x, t) \leq \kappa_{2}|x|^{p \alpha}, \\
& \frac{\partial^{\alpha}}{\partial t^{\alpha}} V(x, t) \leq-\kappa_{3}|x|^{p \alpha},
\end{aligned}
$$


for certain positive constants $\kappa_{1}, \kappa_{2}, \kappa_{3}$ where $f$ and $q$ are arbitrary functions.

Proof. Displaying the difference $V(x(t), t)-V(x, s)$ in terms of the following integral equation

$$
V(x(t), t)-V(x, s)=\frac{1}{\Gamma(\alpha+1)} \int_{s}^{t} \frac{\partial^{\alpha}}{\partial t^{\alpha}} V(x(u), u)(d u)^{\alpha}
$$

and calculating expectations, we have

$$
\mathbf{E} V(x(t), t)-V(x, s)=\frac{1}{\Gamma(\alpha+1)} \int_{s}^{t} \mathbf{E} \frac{\partial^{\alpha}}{\partial t^{\alpha}} V(x(u), u)(d u)^{\alpha} .
$$

Differentiating with respect to $t^{\alpha}$ and employing (2.7) and (2.8), we obtain

$$
\frac{\partial^{\alpha}}{\partial t^{\alpha}} \mathbf{E} V(x(t), t) \leq-\frac{\kappa_{3}}{\kappa_{2}} \mathbf{E} V(x(t), t) .
$$

Hence

$$
\mathbf{E} V(x(t), t) \leq V(x, t) E_{\alpha}\left(-\frac{\kappa_{3}}{\kappa_{2}}(t-s)^{\alpha}\right) .
$$

This together with (2.7) yields (4.2) and the proof is complete.

\section{Stochastic processes as solutions of fractional differen- tial equations}

Let $\mathbf{L}$ denotes the class of functions such that on every finite interval $[0, T]$, and

$$
\frac{1}{\Gamma(\alpha+1)} \int_{0}^{T}|f(t)|(d t)^{\alpha}<\infty, \quad 0<\alpha \leq 1 .
$$

Moreover, assume $\xi(t, \omega)(t \geq 0)$ denotes a separable measurable stochastic process with values in $\mathbb{R}^{k}$, and $F(x, t, z)\left(x \in \mathbb{R}^{l}, t \geq 0, z \in \mathbb{R}^{k}\right)$ denotes a Borel-measurable function of $(x, t, z)$ which fulfills the following assumptions: 1. The process $F(0, t, \xi(t, \omega))$ is in $\mathbf{L}$, namely, for all $T>0$

$$
\mathbf{P}\left\{\frac{1}{\Gamma(\alpha+1)} \int_{0}^{T}|F(0, t, \xi(t, \omega))|(d t)^{\alpha}<\infty\right\}=1, \quad 0<\alpha \leq 1,
$$

where $\mathbf{P}$ is probability measure.

2. There is a stochastic process $B(t, \omega) \in \mathbf{L}$ such that for each $x_{i} \in \mathbb{R}^{l}$

$$
\left|F\left(x_{1}, t, \xi\left(t, \omega_{1}\right)\right)-F\left(x_{2}, t, \xi\left(t, \omega_{2}\right)\right)\right| \leq B(t, \omega)\left|x_{1}-x_{2}\right| .
$$

We will say that a function $x(t, \omega)$ is a solution of the equation

$$
\frac{d^{\alpha} x}{d t^{\alpha}}=F(x, t, \xi(t, \omega)), \quad 0<\alpha \leq 1
$$

with initial condition

$$
x\left(t_{0}, \omega\right)=x_{0}(\omega)
$$


on the interval $\left[t_{0}, t_{1}\right]$ if for all $t \in\left[t_{0}, t_{1}\right]$

$$
x(t, \omega)=x_{0}(\omega)+\frac{1}{\Gamma(\alpha+1)} \int_{t_{0}}^{t} F(x, s, \xi(s, \omega))(d s)^{\alpha} .
$$

Moreover, we will show that under the assumptions (3.1) and (3.2) these equations determine a new stochastic process in $\mathbb{R}^{l}$ for $t \geq t_{0}$.

Theorem 2. Under the assumptions (3.1) and (3.2), the problem (3.3)-(3.4) has a unique solution $x(t, \omega)$, which determines a stochastic process. This solution is almost absolutely fractional continuous for all $t \geq t_{0}$ and satisfies the following estimate

$$
\begin{aligned}
\left|x(t, \omega)-x_{0}(t, \omega)\right| \leq & \frac{1}{\Gamma(\alpha+1)} \int_{t_{0}}^{t}\left|F\left(x_{0}(\omega), s, \xi(s, \omega)\right)\right|(d s)^{\alpha} \\
& \times E_{\alpha}\left(\int_{t_{0}}^{t} B(s, \omega)(d s)^{\alpha}\right) .
\end{aligned}
$$

Proof. We can detect numbers $R$ and $t_{1}>t_{0}$ such that $\left|x_{0}(\omega)\right| \leq \frac{R}{2}$ and

$$
\begin{aligned}
\phi\left(t_{0}, t_{1}, \omega\right)= & \frac{1}{\Gamma(\alpha+1)} \int_{t_{0}}^{t_{1}}\left|F\left(x_{0}(\omega), s, \xi(s, \omega)\right)\right|(d s)^{\alpha} \\
& \times E_{\alpha}\left(\int_{t_{0}}^{t_{1}} B(s, \omega)(d s)^{\alpha}\right)=\frac{R}{2} .
\end{aligned}
$$

Utilizing the method of successive approximations [23] to (3.5) on the interval $\left[t_{0}, t_{1}\right]$

$$
\begin{aligned}
x^{(n+1)}(t, \omega) & =x_{0}(\omega)+\frac{1}{\Gamma(\alpha+1)} \int_{t_{0}}^{t} F\left(x^{n}(s, \omega), s, \xi(s, \omega)\right)(d s)^{\alpha}, \\
x^{0}(t, \omega) & \equiv x_{0}(\omega),
\end{aligned}
$$

and employing (3.1), (3.2) and (3.6), we have the following estimates

$$
\begin{gathered}
\left|x^{(1)}(t, \omega)-x_{0}(t, \omega)\right| \leq \frac{1}{\Gamma(\alpha+1)} \int_{t_{0}}^{t}\left|F\left(x_{0}(\omega), s, \xi(s, \omega)\right)\right|(d s)^{\alpha} \leq \frac{R}{2}, \\
\ldots \\
\left|x^{(n+1)}(t, \omega)-x^{(n)}(t, \omega)\right| \leq \frac{1}{\Gamma(\alpha+1)} \int_{t_{0}}^{t} B(s, \omega)\left|x^{(n)}(s, \omega)-x^{(n-1)}(s, \omega)\right|(d s)^{\alpha} .
\end{gathered}
$$

These together with (3.6) yield

$$
\begin{aligned}
\left|x^{(n+1)}(t, \omega)-x^{(n)}(t, \omega)\right| \leq & \frac{1}{\Gamma(\alpha+1)} \int_{t_{0}}^{t}\left|F\left(x_{0}(\omega), s, \xi(s, \omega)\right)\right|(d s)^{\alpha} \\
& \times \frac{\left[\int_{t_{0}}^{t} B(s, \omega)(d s)^{\alpha}\right]^{n}}{\Gamma(n \alpha+1)} .
\end{aligned}
$$

It concludes from $(3.7)$ that $\lim _{n \rightarrow \infty} x^{(n)}(t, \omega)$ exists and that it fulfils $(3.5)$. The proof of uniqueness is analogous (i.e., the solution can be unlimitedly continued for $\left.t \geq t_{0}\right)$. 
Let us consider $T>t_{0}$ and select $R$ such that, besides the relations $\left|x_{0}(\omega)\right| \leq$ $\frac{R}{2}$ and (3.6), we also have the estimate inf $\left\{t: t \geq t_{0},|x(t, \omega)|>\frac{R}{2}\right\}>T$. Then, it concludes that $x\left(t_{1}, \omega\right)<\frac{R}{2}$ and therefore the solutions can be continued to a point $t_{2}$ such that $\phi\left(t_{1}, t_{2}, \omega\right)=\frac{R}{2}$. Repeating this manner, we have $t_{n} \geq T$ for some $n$, since the functions $F$ and $B$ are fractional integrable on every finite interval. This completes the proof.

\section{New definition of string stability based on Mittag-Leffler function}

Let us consider the interconnected stochastic system as follows

$$
\frac{d^{\alpha} x^{i}}{d t^{\alpha}}=F\left(x^{i}, \ldots, x^{i-r+1}, t, \xi^{i}(t, \omega)\right), \quad 0<\alpha \leq 1,
$$

where $i \in \mathbb{N}, x^{i} \in \mathbb{R}^{n}, x^{i-j} \equiv 0$ for any $i \leq j$, and $t \in\left[t_{0}, \infty\right)$. Moreover, consider the following vector function

$$
F: \underbrace{\mathbb{R}^{n} \times \ldots \times \mathbb{R}^{n}}_{\mathrm{r} \text { times }} \times \mathbb{R}^{+} \times \mathbb{R}^{l} \rightarrow \mathbb{R}^{n},
$$

such that $F(0, \ldots, 0)=0$ and $\xi^{i}$ be independent stochastic processes from $\mathbb{R}^{l}$. Let for every $t_{0} \in \mathbb{R}^{+}$and every $x_{0}^{i} \in \mathbb{R}^{n}$, equation (4.1) has a unique absolutely fractional continuous solution $x^{i}(t, \omega)$ for $t \geq t_{0}$ via $x^{i}\left(t_{0}, \omega\right)=x_{0}^{i}$. Moreover, suppose that for $j<i$ processes $x^{j}(t, \omega)$ and $\xi^{i}(t, \omega)$ be independent. For the sake of convenience, let $t_{0}=0$. We utilize the following symbolizations: $|\cdot|$ denotes the Euclidean norm, for any $p \alpha<\infty$, where $p \in \mathbb{R}^{+}$and $0<\alpha \leq 1$

$$
\begin{aligned}
& \|f(0)\|_{\infty}^{p \alpha}:=\sup _{i \in \mathbb{N}} \mathbf{E}\left[\left|f^{i}(0)\right|^{p \alpha}\right], \\
& \left\|f^{i}\right\|_{\infty}^{p \alpha}=\left\|f^{i}(\cdot)\right\|_{\infty}^{p \alpha}:=\sup _{t \geq 0} \mathbf{E}\left[\left|f^{i}(t)\right|^{p \alpha}\right],
\end{aligned}
$$

where $\mathbf{E}$ denotes the expectation of stochastic process.

To deduce stability results we define the following definitions.

Definition 1. The origin $x^{i}=0, i \in \mathbb{N}$ of system (4.1) is $(p \alpha)$-mean string stable if given any $\epsilon>0$, there is $\delta>0$ such that:

$$
\|x(0)\|_{\infty}^{p \alpha}<\delta \Longrightarrow \sup _{i \in \mathbb{N}}\left\|x^{i}(\cdot)\right\|_{\infty}^{p \alpha}<\epsilon
$$

Definition 2. The origin $x^{i}=0, i \in \mathbb{N}$ of system (4.1) is Mittag-Leffler string $(p \alpha)$-stable if it is $(p \alpha)$-mean string stable and if there are positive constants $c_{i}$ and $\beta_{i}$, such that

$$
\mathbf{E}\left[\left|x^{i}(t)\right|^{p \alpha}\right]<c_{i}\left|x_{0}^{i}\right|^{p \alpha} E_{\alpha}\left(-\beta_{i}\left(t-t_{0}\right)^{\alpha}\right)
$$

for all $i \in \mathbb{N}$. 
In particular case for $p=1$ and $p=2$ they are called Mittag-Leffler $\alpha$-mean and $\alpha$-mean square string stability, respectively. The case most frequently considered in the literature to date is that of exponentially string $p$-stability for $p=1, \alpha=1$ (exponential stability in the mean string) and for $p=2, \alpha=1$ (exponential stability in mean square string).

Theorem 3. If the origin $x^{i}=0$ of the system (2.6) is Mittag-Leffler ( $\left.p \alpha\right)$ stable and $f$ and $q$ have bounded fractional derivatives with respect to $x^{i^{p \alpha}}$, then there is a function $V\left(x^{i}, t\right) \in \mathbf{C}_{\alpha}^{0}(E)$ which fulfils the inequalities (2.7) and (2.8) and also

$$
\left|\frac{\partial^{\alpha} V}{\partial x^{i^{\alpha}}}\right|<\kappa_{4}\left|x^{i}\right|^{(p-1) \alpha}
$$

Proof. We assert that the function

$$
V\left(x^{i}, t\right)=\frac{1}{\Gamma(\alpha+1)} \int_{t}^{t+T} \mathbf{E}\left|x^{i}(u)\right|^{p \alpha}(d u)^{\alpha}
$$

fulfils all the conditions of theorem for appropriate constant $T>0$. Simply, by $(4.2)$

$$
V\left(x^{i}, t\right) \leq \frac{1}{\Gamma(\alpha+1)} \int_{t}^{t+T} c\left|x^{i}\right|^{p \alpha} E_{\alpha}\left(-\beta_{i}\left(t-t_{0}\right)^{\alpha}\right)(d u)^{\alpha}=k_{1}\left|x^{i}\right|^{p \alpha} .
$$

Since the coefficients $f$ and $q$ have bounded fractional derivatives with respect to $x^{i^{p \alpha}}$, we have

$$
\left|f\left(x^{i}, t\right)\right|<\kappa_{5}\left|x^{i}\right|^{p \alpha}, \quad\left|q\left(x^{i}, t\right)\right|<\kappa_{5}\left|x^{i}\right|^{p \alpha},
$$

and therefore

$$
\left|\frac{d^{\alpha}}{d t^{\alpha}}\left(\left|x^{i}\right|^{p \alpha}\right)\right| \leq \kappa_{6}\left|x^{i}\right|^{p \alpha}
$$

Employing the integral equation as formula (2.9) to the function $\left|x^{i}\right|^{p \alpha}$ and using (4.3), we obtain

$$
\begin{aligned}
& \mathbf{E}\left|x^{i}(t+T)\right|^{p \alpha}-\left|x^{i}\right|^{p \alpha}=\frac{1}{\Gamma(\alpha+1)} \int_{t}^{t+T} \mathbf{E} \frac{d^{\alpha}}{d t^{\alpha}}\left(\left|x^{i}(u)\right|^{p \alpha}\right)(d u)^{\alpha} \\
& \geq-\frac{1}{\Gamma(\alpha+1)} \kappa_{6} \int_{t}^{t+T} \mathbf{E}\left|x^{i}(u)\right|^{p \alpha}(d u)^{\alpha}=-\kappa_{6} V\left(t, x^{i}\right) .
\end{aligned}
$$

We choose $T$ so that

$$
\mathbf{E}\left|x^{i}(t+T)\right|^{p \alpha} \leq \frac{1}{2}\left|x^{i}\right|^{p \alpha}
$$

and hence, we have the inequality $V\left(x^{i}, t\right) \geq \frac{\left|x^{i}\right|^{p \alpha}}{\left(2 \kappa_{6}\right)}$. This proves (2.7). To prove the required smoothness of $V\left(x^{i}, t\right)$ and to verify (2.8), we utilize (4.4) and deduce the following estimate

$$
\begin{aligned}
& \left|\frac{\partial^{\alpha}}{\partial x^{i^{\alpha}}} V\left(x^{i}, t\right)\right|=\left.\left|\frac{1}{\Gamma(\alpha+1)} \int_{t}^{t+T} \frac{\partial^{\alpha}}{\partial x^{i^{\alpha}}} \mathbf{E}\right| x^{i}(u)\right|^{p \alpha}(d u)^{\alpha} \mid \\
& \leq \frac{1}{\Gamma(\alpha+1)} \int_{t}^{t+T} \kappa_{1}\left|x^{i}\right|^{(p-1) \alpha} E_{\alpha}\left(\kappa_{2}(u-t)^{\alpha}\right)(d u)^{\alpha}=\kappa_{4}\left|x^{i}\right|^{(p-1) \alpha} .
\end{aligned}
$$




\section{Estimation of a certain Mittag-Leffler functional of a Gaussian process}

The following evaluation plays a fundamental role in the theory of stability of fractional stochastic systems:

$$
\mathbf{E}\left[E_{\alpha}\left(k_{1} \int_{s}^{t}\left|\xi_{1}^{i}(\tau)\right|(d \tau)^{\alpha}\right)\right] \leq E_{\alpha}\left(k_{2}\left(t^{\alpha}-s^{\alpha}\right)\right), \quad 0<\alpha \leq 1, \quad t \geq s .
$$

Stability of the system under random perturbations needs that for each $\epsilon>0$ there exists a $\gamma>0$ such that for $t>s$

$$
\mathbf{E}\left[E_{\alpha}\left(\gamma \int_{s}^{t}\left|\xi_{1}^{i}(\tau)\right|(d \tau)^{\alpha}\right)\right] \leq E_{\alpha}\left(\epsilon\left(t^{\alpha}-s^{\alpha}\right)\right)
$$

In this section we will obtain straightforward conditions for these evaluations to hold for Gaussian processes.

We will consider a Gaussian stochastic process $\xi(t, \omega) \in \mathbb{R}^{l}$, namely, a process which its finite-dimensional distributions are Gaussian. Moreover, assume that this process be measurable and its kernel $K(s, t)=\operatorname{cov}(\xi(s), \xi(t))$ be continuous.

Lemma 3. The process $\xi(t, \omega), t_{0} \leq t \leq t_{1}$, can be expressed by the following series

$$
\xi(t, \omega)=\sum_{i=1}^{\infty} \sqrt{\lambda_{i}} \phi_{i}(t) \xi_{i}(\omega)
$$

This series is almost surely convergent for every $t$ and fulfills the Parseval's identity in the scope of local fractional calculus [16, 28, 29,31]

$$
\int_{t_{0}}^{t_{1}}|\xi(t, \omega)|^{2}(d t)^{\alpha}=\sum_{i=1}^{\infty} \lambda_{i} \xi_{i}^{2}
$$

Here $\phi_{i}(t)$ and $\lambda_{i}$ are the normalized eigenfunctions and eigenvalues of the following integral equation

$$
\int_{t_{0}}^{t_{1}} K(t, \tau) \phi(\tau)(d \tau)^{\alpha}=\lambda \phi(t)
$$

and $\xi_{i}$ are independent Gaussian random variables with unit variance and zero expectation.

Proof. The proof follows from the expression of the process $\xi(t, \omega)$ in terms of eigenfunctions of (5.2). The following formulas for the fractional Fourier coefficients

$$
\sqrt{\lambda_{i}} \xi_{i}(\omega)=\int_{t_{0}}^{t_{1}} \xi(t, \omega) \phi_{i}(t)(d t)^{\alpha}
$$

the orthogonality of the $\phi_{i}(t)$ and the truth that the process is Gaussian indicate that the random variables $\xi_{i}$ are independent. Relation (5.1) follows from the completeness of the system of eigenfunctions $\phi_{i}(t)$. (For more details see, e.g., [20] and [9], Chap. 5, Sect. 2, which deal with the integer order case $\alpha=1)$. 
Lemma 4. The expectation of the following functional exists for all $t_{0}<t_{1}$ and for sufficiently small positive $\nu$

$$
\mathbf{E} E_{\alpha}\left(\nu \int_{t_{0}}^{t_{1}}|\xi(t, \omega)|^{2}(d t)^{\alpha}\right) .
$$

In addition, we have the representation

$$
\mathbf{E} E_{\alpha}\left(\nu \int_{t_{0}}^{t_{1}}|\xi(t, \omega)|^{2}(d t)^{\alpha}\right)=\prod_{i=1}^{\infty} \frac{1}{\sqrt{1-\frac{2 \nu \lambda_{i}}{\Gamma(\alpha+1)}}} .
$$

If also the Gaussian process $\xi(t, \omega)$ with zero expectation fulfils

$$
\begin{aligned}
\operatorname{trace} K(s, s) & =\mathbf{E}|\xi(s, \omega)|^{2} \leq c, \\
\int_{0}^{\infty}\|K(s, u)\|(d u)^{\alpha} & =\int_{0}^{\infty}\|K(u, s)\|(d u)^{\alpha} \leq c^{\prime},
\end{aligned}
$$

for some $c, c^{\prime}>0$ and all $s>0$, then for all $t_{0}<t_{1}$

$$
\mathbf{E} E_{\alpha}\left(\nu \int_{t_{0}}^{t_{1}}|\xi(t, \omega)|^{2}(d t)^{\alpha}\right) \leq E_{\alpha}\left(\frac{\nu c}{1-\frac{2 \nu c^{\prime}}{\Gamma(\alpha+1)}}\left(t_{1}^{\alpha}-t_{0}^{\alpha}\right)\right) .
$$

Proof. Using (5.1) and (5.4), we have

$$
\sum_{i=1}^{\infty} \lambda_{i}=\mathbf{E} \int_{t_{0}}^{t_{1}}|\xi(u, \omega)|^{2}(d u)^{\alpha} \leq c\left(t_{1}^{\alpha}-t_{0}^{\alpha}\right)
$$

Hence, $\lambda_{\max }=\max _{1 \leq i \leq \infty} \lambda_{i}$ exists. Without loss of generality let $\lambda_{\max }=\lambda_{1}$. Relation (5.3) follows from (5.1) and the following estimate, valid for $\nu<$ $\Gamma(\alpha+1) /\left(2 \lambda_{1}\right)$ :

$$
\mathbf{E} E_{\alpha}\left(\nu \lambda_{i} \xi_{i}^{2}(\omega)\right)=\frac{1}{\sqrt{1-2 \nu \lambda_{i} / \Gamma(\alpha+1)}} .
$$

We now prove that $\lambda_{1}=\lambda_{\max } \leq c^{\prime}$. Indeed, we can deduce from (5.5) that

$$
\begin{aligned}
\lambda_{1} & =\int_{t_{0}}^{t_{1}} \lambda_{1}\left(\phi_{1}(s), \phi_{1}(s)\right)(d s)^{\alpha}=\int_{0}^{t_{1}} \int_{0}^{t_{1}}\left(K(s, t) \phi_{1}(t), \phi_{1}(s)\right)(d s)^{\alpha}(d t)^{\alpha} \\
& \leq \int_{0}^{t_{1}} \int_{0}^{t_{1}}\|K(s, t)\|\left|\phi_{1}(t)\right|\left|\phi_{1}(s)\right|(d s)^{\alpha}(d t)^{\alpha} \\
& \leq \int_{0}^{t_{1}} \int_{0}^{t_{1}}\|K(s, t)\| \frac{\left|\phi_{1}(t)\right|^{2}+\left|\phi_{1}(s)\right|^{2}}{2}(d s)^{\alpha}(d t)^{\alpha} \leq c^{\prime}
\end{aligned}
$$

Using the primary relation $1+\frac{\gamma}{\Gamma(\alpha+1)}<E_{\alpha}(\gamma)$ for $\gamma>0$, we obtain the relation

$$
\begin{aligned}
& \prod_{i=1}^{\infty} \frac{1}{\sqrt{1-\frac{2 \nu \lambda_{i}}{\Gamma(\alpha+1)}}}=\prod_{i=1}^{\infty}\left(1+\frac{2 \nu \lambda_{i}}{\Gamma(\alpha+1)}+\frac{\frac{4 \nu^{2} \lambda_{i}^{2}}{\Gamma(\alpha+1)^{2}}}{1-\frac{2 \nu \lambda_{i}}{\Gamma(\alpha+1)}}\right)^{1 / 2} \\
& \leq E_{\alpha}\left(\nu\left(1+\frac{\frac{2 \nu \lambda_{1}}{\Gamma(\alpha+1)}}{1-\frac{2 \nu \lambda_{1}}{\Gamma(\alpha+1)}}\right) \sum_{i=1}^{\infty} \lambda_{i}\right)=E_{\alpha}\left(\frac{\nu}{1-\frac{2 \nu \lambda_{1}}{\Gamma(\alpha+1)}} \sum_{i=1}^{\infty} \lambda_{i}\right) .
\end{aligned}
$$

Hence, by use of (5.7) and (5.8), we obtain (5.6). 
Theorem 4. Suppose that the Gaussian process $\xi(t, \omega)$ with zero expectation fulfills (5.4) and (5.5). Then the following inequality holds:

$$
\mathbf{E} E_{\alpha}\left(k_{1} \int_{t_{0}}^{t_{1}}|\xi(t, \omega)|(d s)^{\alpha}\right) \leq E_{\alpha}\left(k_{1}\left(\sqrt{c}+\frac{k_{1} c^{\prime}}{2 \Gamma(\alpha+1)}\right)\left(t_{1}^{\alpha}-t_{0}^{\alpha}\right)\right) .
$$

Proof. Using the following inequality $a \leq \frac{\nu}{2} a^{2}+\frac{1}{2 \nu}, \nu>0$, we obtain

$$
\mathbf{E} E_{\alpha}\left(k_{1} \int_{t_{0}}^{t_{1}}|\xi(s, \omega)|(d s)^{\alpha}\right) \leq E_{\alpha}\left(\frac{k_{1}}{2 \nu}\left(t_{1}^{\alpha}-t_{0}^{\alpha}\right)\right) \mathbf{E} E_{\alpha}\left(\frac{k_{1} \nu}{2} \int_{t_{0}}^{t_{1}}|\xi(s, \omega)|^{2}(d s)^{\alpha}\right) .
$$

Therefore, it concludes from Lemma 4 that for all $\nu<\frac{\Gamma(\alpha+1)}{k_{1} c^{\prime}}$

$$
\mathbf{E} E_{\alpha}\left(k_{1} \int_{t_{0}}^{t_{1}}|\xi(s, \omega)|(d s)^{\alpha}\right) \leq E_{\alpha}\left(\left[\frac{k_{1}}{2 \nu}+\frac{k_{1} \nu c}{2\left(1-\frac{\nu k_{1} c^{\prime}}{\Gamma(\alpha+1)}\right)}\right]\left(t_{1}^{\alpha}-t_{0}^{\alpha}\right)\right) .
$$

Setting $\nu=\nu^{*}=1 /\left(\frac{k_{1} c^{\prime}}{\Gamma(\alpha+1)}+\sqrt{c}\right)$, we obtain $(5.9)$.

Lemma 5. Assume that $U_{i}(t) \geq 0$ for all $t \geq 0, i \in \mathbb{N}$ and

$$
\frac{d^{\alpha} U_{i}}{d t^{\alpha}} \leq-\theta_{0} U_{i}+\gamma_{0}\left|\xi_{1}^{i}(t)\right| U_{i}+\beta_{0}\left|\xi_{2}^{i}(t)\right| U_{i}+\sum_{j=2}^{\infty} \theta_{j} U_{i-j+1},
$$

where $U_{-j} \equiv 0$ and the real constants $\theta_{0}, \gamma_{0}, \beta_{0}, \theta_{j}$ be positive for $j \in \mathbb{N}$, and for $0 \leq s<t$

$$
\left\{\begin{array}{l}
\mathbf{E}\left[E_{\alpha}\left(2 \gamma_{0} \int_{s}^{t}\left|\xi_{1}^{i}(\tau)\right|(d \tau)^{\alpha}\right)\right] \leq E_{\alpha}\left(2 \gamma_{0} s_{1}\left(t^{\alpha}-s^{\alpha}\right)\right), \quad 0<\alpha \leq 1 \\
\mathbf{E}\left[E_{\alpha}\left(2 \beta_{0} \int_{s}^{t}\left|\xi_{2}^{i}(\tau)\right|(d \tau)^{\alpha}\right)\right] \leq E_{\alpha}\left(2 \beta_{0} s_{2}\left(t^{\alpha}-s^{\alpha}\right)\right), \quad 0<\alpha \leq 1 \\
\theta=\min \left\{\theta_{0}-2 \beta_{0} s_{2}, \theta_{0}-2 \gamma_{0} s_{1}\right\}>\sum_{j=2}^{\infty} \theta_{j} \geq 0
\end{array}\right.
$$

Moreover, $U_{j}(t), \xi_{1}^{i}(t)$ and $U_{j}(t), \xi_{2}^{i}(t)$ be independent processes for $j<i$, $i \in \mathbb{N}$. Then, for each $\epsilon>0$ there is $\delta>0$ such that

$$
\|U(0)\|_{\infty}^{1}<\delta \Rightarrow \sup _{i \in \mathbb{N}}\left\|U_{i}\right\|_{\infty}^{1}<\epsilon
$$

and $\mathbf{E}\left[U_{i}\right] \rightarrow 0$ for $t \rightarrow \infty$.

Proof. Considering the condition (5.10) and Lemma 1 we have

$$
\begin{aligned}
U_{i}(t) \leq & U_{i}(0) E_{\alpha}\left(-\theta_{0} t^{\alpha}\right) E_{\alpha}\left(\int_{0}^{t} \gamma_{0}\left|\xi_{1}^{i}(s)\right|(d s)^{\alpha}\right) E_{\alpha}\left(\int_{0}^{t} \beta_{0}\left|\xi_{2}^{i}(s)\right|(d s)^{\alpha}\right) \\
& +\int_{0}^{t} E_{\alpha, \alpha}\left(-\theta_{0}\left(t^{\alpha}-s^{\alpha}\right)\right) E_{\alpha, \alpha}\left(\int_{s}^{t} \gamma_{0}\left|\xi_{1}^{i}(\tau)\right|(d \tau)^{\alpha}\right) \\
& \times E_{\alpha, \alpha}\left(\int_{s}^{t} \beta_{0}\left|\xi_{2}^{i}(\tau)\right|(d \tau)^{\alpha}\right) \cdot \sum_{j=2}^{\infty} \theta_{j} U_{i-j+1}(d s)^{\alpha} .
\end{aligned}
$$


The above relation together with $\mu \cdot \nu \leq \frac{\mu^{2}+\nu^{2}}{2}$, and the semigroup properties (2.3) conclude that

$$
\begin{aligned}
U_{i}(t) & \leq U_{i}(0) E_{\alpha}\left(-\theta_{0} t^{\alpha}\right)\left[\frac{1}{2} E_{\alpha}\left(2 \int_{0}^{t} \gamma_{0}\left|\xi_{1}^{i}(s)\right|(d s)^{\alpha}\right)\right. \\
& \left.+\frac{1}{2} E_{\alpha}\left(2 \int_{0}^{t} \beta_{0}\left|\xi_{2}^{i}(s)\right|(d s)^{\alpha}\right)\right] \\
& +\int_{0}^{t} E_{\alpha, \alpha}\left(-\theta_{0}(t-s)^{\alpha}\right)\left[\frac{1}{2} E_{\alpha, \alpha}\left(2 \int_{s}^{t} \gamma_{0}\left|\xi_{1}^{i}(\tau)\right|(d \tau)^{\alpha}\right)\right. \\
& \left.+\frac{1}{2} E_{\alpha, \alpha}\left(2 \int_{s}^{t} \beta_{0}\left|\xi_{2}^{i}(\tau)\right|(d \tau)^{\alpha}\right)\right] \cdot \sum_{j=2}^{\infty} \theta_{j} U_{i-j+1} d s .
\end{aligned}
$$

Therefore

$$
\begin{aligned}
& \mathbf{E}\left[U_{i}(t)\right] \leq \frac{1}{2} \mathbf{E}\left[U_{i}(0)\right] E_{\alpha}\left(\left(-\theta_{0}+2 \gamma_{0} s_{1}\right) t^{\alpha}\right)+\frac{1}{2} \mathbf{E}\left[U_{i}(0)\right] E_{\alpha}\left(\left(-\theta_{0}+2 \beta_{0} s_{2}\right) t^{\alpha}\right) \\
& +\frac{1}{2} \int_{0}^{t} E_{\alpha, \alpha}\left(\left(-\theta_{0}+2 \gamma_{0} s_{1}\right)(t-s)^{\alpha}\right) \sum_{j=2}^{\infty} \theta_{j} \mathbf{E}\left[U_{i-j+1}\right](d s)^{\alpha} \\
& +\frac{1}{2} \int_{0}^{t} E_{\alpha, \alpha}\left(\left(-\theta_{0}+2 \beta_{0} s_{2}\right)(t-s)^{\alpha}\right) \sum_{j=2}^{\infty} \theta_{j} \mathbf{E}\left[U_{i-j+1}\right](d s)^{\alpha}
\end{aligned}
$$

Having employed the fractional integration rule of the Mittag-Leffler function (see relation (2.9) of [23]), along with some manipulation (relation (2.8) of [23]), concludes that

$$
\sup _{t \geq 0} \mathbf{E}\left[U_{i}(t)\right] \leq \mathbf{E}\left[U_{i}(0)\right]+\frac{1}{2} \sum_{j=2}^{\infty} \theta_{j} \sup _{t \geq 0} \mathbf{E}\left[U_{i-j+1}(t)\right]\left\{\frac{1}{\theta_{0}-2 \beta_{0} s_{2}}+\frac{1}{\theta_{0}-2 \gamma_{0} s_{1}}\right\} \text {. }
$$

Assume that $\theta=\min \left\{\theta_{0}-2 \beta_{0} s_{2}, \theta_{0}-2 \gamma_{0} s_{1}\right\}$. Then, for each $i \in \mathbb{N}$ we have

$$
\left\|U_{i}\right\|_{\infty}^{1} \leq \mathbf{E}\left[U_{i}(0)\right]+\sum_{j=2}^{\infty} \frac{\theta_{j}}{\theta}\left\|U_{i-j+1}\right\|_{\infty}^{1} .
$$

To prove (5.12), we need only to indicate that

$$
\left\|U_{i}\right\|_{\infty}^{1} \leq \Theta\|U(0)\|_{\infty}^{1}, \quad \Theta=\theta /\left(\theta-\sum_{j=2}^{\infty} \theta_{j}\right)>1 .
$$

We indicate this relation by induction. For $i=1$, we obtain from (5.14) that:

$$
\left\|U_{i}\right\|_{\infty}^{1} \leq \mathbf{E}\left[U_{i}(0)\right]
$$

To suppose that the hypothesis be correct for the integer $i$, we get

$$
\begin{aligned}
\left\|U_{i+1}(t)\right\|_{\infty}^{1} \leq & \mathbf{E}\left[U_{i+1}(0)\right]+\sum_{j=2}^{\infty} \frac{\theta_{j}}{\theta}\|U(0)\|_{\infty}^{1} \\
& \leq\left(1+\Theta \sum_{j=2}^{\infty} \frac{\theta_{j}}{\theta}\right)\|U(0)\|_{\infty}^{1}=\Theta\|U(0)\|_{\infty}^{1} .
\end{aligned}
$$


Now we prove that $\mathbf{E}\left[U_{i}\right] \rightarrow 0$. This, also is shown by induction. For $i=1$, we obtain from (5.13) that

$$
\mathbf{E}\left[U_{1}(t)\right] \leq \mathbf{E}\left[U_{1}(0)\right] E_{\alpha}\left(-\theta t^{\alpha}\right),
$$

where $\theta$ is defined by (5.11). Let the hypothesis be correct for each $j<i$

$$
\mathbf{E}\left[U_{j}(t)\right] \leq \beta_{j} \mathbf{E}\left[U_{j}(0)\right] E_{\alpha}\left(-\gamma_{j} t^{\alpha}\right)
$$

where the constants $\beta_{j}, \gamma_{j}$ are positive.

Assume that $\beta=\max _{j<i}\left(\beta_{j} \mathbf{E}\left[U_{j}(0)\right]\right)$ and $\gamma=\min _{j<i} \gamma_{j}$, then

$$
\sum_{j=2}^{\infty} \theta_{j} \mathbf{E}\left[U_{i-j+1}(t)\right] \leq \beta E_{\alpha}\left(-\gamma t^{\alpha}\right) \sum_{j=2}^{\infty} \theta_{j}
$$

Applying (5.13) and (5.15) we find

$\mathbf{E}\left[U_{i}(t)\right] \leq \mathbf{E}\left[U_{i}(0)\right] E_{\alpha}\left(-k t^{\alpha}\right)+\int_{0}^{t} E_{\alpha, \alpha}\left(-k(t-s)^{\alpha}\right) \sum_{j=2}^{\infty} \theta_{j} \beta E_{\alpha}\left(-k s^{\alpha}\right)(d s)^{\alpha}$,

where $k=\min \{\gamma, \theta\}$. Again, employing the fractional integration rule of the Mittag-Leffler function (Theorem 11.2 of [11]), with some manipulation (relation (11.4) of [11]), concludes that

$$
\mathbf{E}\left[U_{i}(t)\right] \leq k_{0} \mathbf{E}\left[U_{i}(0)\right] E_{\alpha}\left(-k t^{\alpha}\right), \quad k_{0}=\left(1+\beta \sum_{j=2}^{\infty} \theta_{j}\right)
$$

which completes the proof.

\section{Description of singularly perturbed stochastic system of fractional order}

Consider the following singularly perturbed stochastic system of fractional order:

$$
\begin{cases}\frac{d^{\alpha} x^{i}}{d t^{\alpha}} & =F\left(x^{i}, y^{i}, x^{i-1}, \ldots, x^{i-r+1}\right)+Q_{1}\left(x^{i}, y^{i}\right) \xi_{1}^{i}(t), \quad 0<\alpha \leq 1 \\ \epsilon \frac{d^{\alpha} y^{i}}{d t^{\alpha}} & =G\left(x^{i}, y^{i}\right)+\epsilon Q_{2}\left(x^{i}, y^{i}\right) \xi_{2}^{i}(t)\end{cases}
$$

where $i \in \mathbb{N}, x^{i} \in \mathbb{R}^{n}, x^{i-j} \equiv 0$ for any $i \leq j, t \in \mathbb{R}^{+}, y^{i} \in \mathbb{R}^{m}$ and $\epsilon>0$ is the parameter of singular perturbation. Moreover, $F, G$ are nonlinear vector functions, $Q_{1}, Q_{2}$ are matrices, and $\xi_{1}^{i}, \xi_{2}^{i}$ are independent processes.

Now, we introduce the following conditions:

Assumption 1. The equation $G\left(x^{i}, y^{i}\right)=0$ has a unique continuously fractional differentiable solution $y^{i}=\zeta\left(x^{i}\right)$, such that $\zeta(0)=0$. 
This condition represents the reduced system by inserting $y^{i}=\zeta\left(x^{i}\right)$ in (6.1) as

$$
\frac{d^{\alpha} x^{i}}{d t^{\alpha}}=F\left(x^{i}, \zeta\left(x^{i}\right), x^{i-1}, \ldots, x^{i-r+1}\right)+Q_{1}\left(x^{i}, \zeta\left(x^{i}\right)\right) \xi_{1}^{i}(t)
$$

We define the boundary layer state by a new variable $\chi^{i}=y^{i}-\zeta\left(x^{i}\right)$. Then, the full order interconnected system can be presented as follows

$$
\left\{\begin{aligned}
\frac{d^{\alpha} x^{i}}{d t^{\alpha}}= & \widehat{F}\left(x^{i}, \chi^{i}, x^{i-1}, \ldots, x^{i-r+1}\right)+\widehat{Q}_{1}\left(x^{i}, \chi^{i}\right) \xi_{1}^{i}(t) \\
\epsilon \frac{d^{\alpha} y^{i}}{d t^{\alpha}}= & \widehat{G}\left(\epsilon, x^{i}, \chi^{i}, x^{i-1}, \ldots, x^{i-r+1}\right)+\widehat{Q}_{2}\left(\epsilon, x^{i}, \chi^{i}\right) \xi_{1}^{i}(t) \\
& +\widetilde{Q}_{2}\left(\epsilon, x^{i}, \chi^{i}\right) \xi_{2}^{i}(t)
\end{aligned}\right.
$$

where ingredients of $\widehat{F}, \widehat{G}, \widehat{Q}_{1}, \widehat{Q}_{2}$ and $\widetilde{Q}_{2}$ have the following features

$$
\left\{\begin{array}{l}
\widehat{F}_{j}\left(x^{i}, \chi^{i}, x^{i-1}, \ldots, x^{i-r+1}\right)=F_{j}\left(x^{i}, \chi^{i}+\zeta\left(x^{i}\right), x^{i-1}, \ldots, x^{i-r+1}\right) \\
\widehat{Q}_{1_{l k}}\left(x^{i}, \chi^{i}\right)=Q_{1_{l k}}\left(x^{i}, \chi^{i}+\zeta\left(x^{i}\right)\right) \\
\widehat{G}_{l}\left(\epsilon, x^{i}, \chi^{i}, x^{i-1}, \ldots, x^{i-r+1}\right)=G_{l}\left(x^{i}, \chi^{i}+\zeta\left(x^{i}\right)\right) \\
-\epsilon \sum_{j=1}^{n} \frac{\partial \zeta_{l}}{\partial x_{j}^{i}} F_{j}\left(x^{i}, \chi^{i}+\zeta\left(x^{i}\right), x^{i-1}, \ldots, x^{i-r+1}\right) \\
\widehat{Q}_{2_{l k}}\left(\epsilon, x^{i}, \chi^{i}\right)=-\epsilon \sum_{j=1}^{n} \frac{\partial \zeta_{l}}{\partial x_{j}^{i}} Q_{1_{k j}}\left(x^{i}, \chi^{i}+\zeta\left(x^{i}\right)\right) \\
\widetilde{Q}_{2_{l k}}\left(\epsilon, x^{i}, \chi^{i}\right)=\epsilon Q_{2_{l k}}\left(x^{i}, \chi^{i}+\zeta\left(x^{i}\right)\right)
\end{array}\right.
$$

System (6.2) is handled by an interconnection of the following isolated subsystems

$$
\begin{cases}\frac{d^{\alpha} x^{i}}{d t^{\alpha}} & =\widehat{F}\left(x^{i}, \chi^{i}, 0, \ldots, 0\right)+\widehat{Q}_{1}\left(x^{i}, \chi^{i}\right) \xi_{1}^{i}(t) \\ \epsilon \frac{d^{\alpha} y^{i}}{d t^{\alpha}} & =\widehat{G}\left(\epsilon, x^{i}, \chi^{i}, 0, \ldots, 0\right)+\widehat{Q}_{2}\left(\epsilon, x^{i}, \chi^{i}\right) \xi_{1}^{i}(t)+\widetilde{Q}_{2}\left(\epsilon, x^{i}, \chi^{i}\right) \xi_{2}^{i}(t) .\end{cases}
$$

Assumption 2. The reduced order system is Mittag-Leffler $\alpha$-mean square string stable, i.e., there are positive definite functions $U_{i}=U\left(x^{i}\right), i \in \mathbb{N}$ and there are positive constants $\beta_{l}, \beta_{h}, \beta_{1}, \beta_{3}, \beta_{4}, \beta_{1 j}, j=1, \ldots, r$, which fulfil the following relations:

$$
\left\{\begin{array}{l}
\beta_{l}\left|x^{i}\right|^{2 \alpha} \leq U\left(x^{i}\right) \leq \beta_{h}\left|x^{i}\right|^{2 \alpha} \\
\frac{\partial^{\alpha} U_{i}}{\partial x^{i \alpha}} F\left(x^{i}, \zeta\left(x^{i}\right), x^{i-1}, \ldots, x^{i-r+1}\right) \leq-\beta_{1}\left|x^{i}\right|^{2 \alpha}+\sum_{j=2}^{r} \beta_{1 j}\left|x^{i-j+1}\right|^{2 \alpha} \\
\left|\frac{\partial^{\alpha} U_{i}}{\partial x^{i \alpha}}\right|<\beta_{3}\left|x^{i}\right|^{\alpha}, \quad\left|\frac{\partial^{\alpha} U_{i}}{\partial x^{i \alpha}} Q_{1}\left(x^{i}, \zeta\left(x^{i}\right)\right)\right| \leq \beta_{4}\left|x^{i}\right|^{2 \alpha}
\end{array}\right.
$$

For more details see [6] and page 353 of [26] .

Assumption 3. The boundary layer system is Mittag-Leffler $\alpha$-mean square string stable, i.e., there are positive definite functions $V_{i}=V\left(x^{i}, \chi^{i}\right), i \in \mathbb{N}$ and there are positive constants $\theta_{l}, \theta_{h}, \beta_{2}, \beta_{5}, \theta_{2}, \eta_{1}, \eta_{2}, \gamma, \gamma_{j}, j=2, \ldots, r$, which 
fulfil the following relations:

$$
\left\{\begin{array}{l}
\theta_{l}\left|\chi^{i}\right|^{2 \alpha} \leq V\left(x^{i}, \chi^{i}\right) \leq \theta_{h}\left|\chi^{i}\right|^{2 \alpha} \\
\frac{\partial^{\alpha} V_{i}}{\partial \chi^{i \alpha}} G\left(x^{i}, \chi^{i}+\zeta\left(x^{i}\right)\right) \leq-\beta_{2}\left|\chi^{i}\right|^{2 \alpha}, \\
\left(\frac{\partial^{\alpha} V_{i}}{\partial x^{i \alpha}}-\frac{\partial^{\alpha} V_{i}}{\partial \chi^{i \alpha}} \frac{\partial^{\alpha} \zeta}{\partial x^{i \alpha}}\right) F\left(x^{i}, \chi^{i}+\zeta\left(x^{i}\right), x^{i-1}, \ldots, x^{i-r+1}\right) \leq \theta_{2}\left|x^{i}\right|^{\alpha}\left|\chi^{i}\right|^{\alpha} \\
\quad+\gamma\left|\chi^{i}\right|^{2 \alpha}+\sum_{j=2}^{r} \gamma_{j}\left|x^{i-j+1}\right|^{2 \alpha}, \quad \gamma_{j}>0, \\
\left|\left(\frac{\partial^{\alpha} V_{i}}{\partial x^{i \alpha}}-\frac{\partial^{\alpha} V_{i}}{\partial \chi^{i \alpha}} \frac{\partial^{\alpha} \zeta}{\partial x^{i \alpha}}\right) Q_{1}\left(x^{i}, \chi^{i}+\zeta\left(x^{i}\right)\right)\right| \leq \eta_{1}\left|x^{i}\right|^{\alpha}\left|\chi^{i}\right|^{\alpha}+\eta_{2}\left|\chi^{i}\right|^{2 \alpha} \\
\left|\frac{\partial^{\alpha} V_{i}}{\partial \chi^{i \alpha}} Q_{2}\left(x^{i}, \chi^{i}+\zeta\left(x^{i}\right)\right)\right| \leq \beta_{5}\left|\chi^{i}\right|^{2 \alpha} .
\end{array}\right.
$$

Assumption 4. Functions $F, Q_{1}$ satisfy the Lipschitz condition in their arguments, namely, there are positive constants $k_{1}^{Q_{1}}, k_{2}^{Q_{2}}, k_{j}^{F}, j=1, \ldots, r+1$, which fulfil the following relations:

$$
\left\{\begin{array}{c}
\left|F\left(x^{i}, y^{i}, x^{i-1}, \ldots, x^{i-r+1}\right)-F\left(\chi^{i}, y, \chi^{i-1}, \ldots, \chi^{i-r+1}\right)\right| \\
\leq \sum_{j=1}^{r} k_{j}^{F}\left|x^{i-j+1}-\chi^{i-j+1}\right|+k_{r+1}^{F}\left|y^{i}-y\right|, \\
\left|Q_{1}\left(x^{i}, y^{i}\right)-Q_{1}\left(\chi^{i}, y\right)\right| \leq k_{1}^{Q_{1}}\left|x^{i}-\chi^{i}\right|+k_{2}^{Q_{1}}\left|y^{i}-y\right| .
\end{array}\right.
$$

Assumption 5. There are positive constants $k_{1}, k_{2}$, such that $\left|\xi_{1}^{i}(t)\right|,\left|\xi_{2}^{i}(t)\right|$ fulfil the following relations for $0 \leq s<t$ :

$$
\left\{\begin{array}{l}
\mathbf{E}\left[E_{\alpha}\left(\int_{s}^{t} \frac{4 \delta}{k \theta_{l}}\left|\xi_{1}^{i}(\tau)\right|(d \tau)^{\alpha}\right)\right] \leq E_{\alpha}\left(\frac{4 \delta}{k \theta_{l}} k_{1}\left(t^{\alpha}-s^{\alpha}\right)\right), \quad 0<\alpha \leq 1 \\
\mathbf{E}\left[E_{\alpha}\left(\int_{s}^{t} \frac{2 \beta_{5}}{\theta_{l}}\left|\xi_{2}^{i}(\tau)\right|(d \tau)^{\alpha}\right)\right] \leq E_{\alpha}\left(\frac{2 \beta_{5}}{\theta_{l}} k_{2}\left(t^{\alpha}-s^{\alpha}\right)\right), \quad 0<\alpha \leq 1
\end{array}\right.
$$

where $k=\min \left\{\frac{\beta_{h}}{\theta_{h}}, \frac{\beta_{l}}{\theta_{l}}\right\}$ and $\delta=\max \left\{\frac{\beta_{4}}{2}+\frac{\beta_{3} k_{2}^{Q_{1}}}{4}, \frac{k \eta_{2}}{2}+\frac{\beta_{3} k_{2}^{Q_{1}}}{4}\right\}$ and

$$
k_{0}=\min \left\{\frac{\beta_{1}}{\beta_{h}}-\frac{4 \delta}{4 \theta_{l}} k_{1}, \frac{\beta_{1}}{\beta_{h}}-\frac{2 \beta_{5}}{\theta_{l}} k_{2}\right\}>0 .
$$

Assumptions 1-5 imply Mittag-Leffler $\alpha$-mean square string stability of every perturbed subsystem (6.3) which will be proved in next section.

\section{Interconnected stochastic systems: sufficient conditions and the Mittag-Leffler string stability}

Theorem 5. Assume that Assumptions 1-5 hold. Then, there exists a positive constant $\bar{\epsilon}$ such that, for each $\epsilon \in(0, \bar{\epsilon})$, the singularly perturbed system (6.3) is Mittag-Leffler $\alpha$-mean square stable.

Proof. We calculate $\frac{d^{\alpha} U}{d t^{\alpha}}$ for system (6.3)

$$
\begin{aligned}
\left.\frac{d^{\alpha} U}{d t^{\alpha}}\right|_{(6.3)}=\frac{\partial^{\alpha} U}{\partial x^{\alpha}}\left(F(x, \chi+\zeta(x))+Q_{1}(x, \chi+\zeta(x)) \xi_{1}(t)\right) \\
=\frac{\partial^{\alpha} U}{\partial x^{\alpha}}\left(F(x, \zeta(x))+\frac{\partial^{\alpha} U}{\partial x^{\alpha}} Q_{1}(x, \zeta(x)) \xi_{1}(t)+\frac{\partial^{\alpha} U}{\partial x^{\alpha}}(F(x, \chi+\zeta(x))\right. \\
\quad-F(x, \zeta(x)))+\frac{\partial^{\alpha} U}{\partial x^{\alpha}}\left(Q_{1}(x, \chi+\zeta(x))-Q_{1}(x, \zeta(x))\right) \xi_{1}(t) .
\end{aligned}
$$


From Assumptions 2 and 4 we conclude that

$$
\left.\frac{d^{\alpha} U}{d t^{\alpha}}\right|_{(6.3)} \leq-\beta_{1}|x|^{2 \alpha}+\beta_{3} k_{2}^{f}|x|^{\alpha}|\chi|^{\alpha}+\left|\xi_{1}(t)\right|\left(\beta_{4}|x|^{2 \alpha}+\beta_{3} k_{2}^{q_{1}}|x|^{\alpha}|\chi|^{\alpha}\right) .
$$

We calculate $\frac{d^{\alpha} V}{d t^{\alpha}}$ for system (6.3)

$$
\begin{aligned}
\left.\frac{d^{\alpha} V}{d t^{\alpha}}\right|_{(6.3)}= & \frac{\partial^{\alpha} V}{\partial x^{\alpha}}\left(F(x, \chi+\zeta(x))+Q_{1}(x, \chi+\zeta(x)) \xi_{1}(t)\right) \\
& +\frac{\partial^{\alpha} V}{\partial \chi^{\alpha}}\left(\frac{1}{\epsilon} G(x, \chi+\zeta(x))-\frac{\partial^{\alpha} \zeta}{\partial x^{\alpha}} F(x, \chi+\zeta(x))\right) \\
& +\frac{\partial^{\alpha} V}{\partial \chi^{\alpha}}\left(-\frac{\partial^{\alpha} \zeta}{\partial x^{\alpha}} Q_{1}(x, \chi+\zeta(x)) \xi_{1}(t)+Q_{2}(x, \chi+\zeta(x)) \xi_{2}(t)\right) \\
= & \left(\frac{\partial^{\alpha} V}{\partial x^{\alpha}}-\frac{\partial^{\alpha} V}{\partial \chi^{\alpha}} \frac{\partial^{\alpha} \zeta}{\partial x^{\alpha}}\right) F(x, \chi+\zeta(x))+\frac{1}{\epsilon} \frac{\partial^{\alpha} V}{\partial \chi^{\alpha}} G(x, \chi+\zeta(x)) \\
& +\left(\frac{\partial^{\alpha} V}{\partial x^{\alpha}} Q_{1}(x, \chi+\zeta(x))-\frac{\partial^{\alpha} V}{\partial \chi^{\alpha}} \frac{\partial^{\alpha} \zeta}{\partial x^{\alpha}} Q_{1}(x, \chi+\zeta(x))\right) \xi_{1}(t) \\
& +\frac{\partial^{\alpha} V}{\partial \chi^{\alpha}} Q_{2}(x, \chi+\zeta(x)) \xi_{2}(t) .
\end{aligned}
$$

Applying Assumptions 3 and 4 we get

$$
\begin{aligned}
\left.\frac{d^{\alpha} V}{d t^{\alpha}}\right|_{(6.3)} \leq & \theta_{2}|x|^{\alpha}|\chi|^{\alpha}+\gamma|\chi|^{2 \alpha}-\frac{\beta_{2}}{\epsilon}|\chi|^{2 \alpha} \\
& +\left|\xi_{1}(t)\right|\left(\eta_{1}|x|^{\alpha}|\chi|^{\alpha}+\eta_{2}|\chi|^{2 \alpha}\right)+\left|\xi_{2}(t)\right| \beta_{5}|\chi|^{2 \alpha} .
\end{aligned}
$$

Consider the function introduced by:

$$
\Xi=\Xi(x(t), \chi(t))=\frac{1}{2}[U(x(t))+k V(x(t), \chi(t))],
$$

where

$$
k=\min \left\{\beta_{h} / \theta_{h}, \beta_{l} / \theta_{l}\right\} .
$$

From Assumptions 2 and 3 we have

$$
\frac{\beta_{l}|x|^{2 \alpha}+k \theta_{l}|\chi|^{2 \alpha}}{2} \leq \Xi \leq \frac{\beta_{h}|x|^{2 \alpha}+k \theta_{h}|\chi|^{2 \alpha}}{2} .
$$

Using (7.3) and (7.4) we obtain

$$
|x|^{2 \alpha}+|\chi|^{2 \alpha} \geq \frac{2 \Xi}{\beta_{h}}, \quad|x|^{2 \alpha}+|\chi|^{2 \alpha} \leq \frac{2 \Xi}{k \theta_{l}} .
$$

We calculate $\frac{d^{\alpha} \Xi}{d t^{\alpha}}$ for system (6.3)

$$
\left.\frac{d^{\alpha} \Xi}{d t^{\alpha}}\right|_{(6.3)}=\frac{1}{2} \frac{d^{\alpha} U}{d t^{\alpha}}+\frac{k}{2} \frac{d^{\alpha} V}{d t^{\alpha}}
$$


From conditions (7.1) and (7.2) we conclude

$$
\begin{aligned}
& \left.\frac{d^{\alpha} \Xi}{d t^{\alpha}}\right|_{(6.3)} \leq-\left(\frac{\beta_{1}}{2}|x|^{2 \alpha}-\frac{\beta_{3} k_{2}^{F}+k \theta_{2}}{2}|x|^{\alpha}|\chi|^{\alpha}+\frac{k}{2}\left(\frac{\beta_{3}}{\epsilon}-\gamma\right)|\chi|^{2 \alpha}\right) \\
& +\left|\xi_{1}(t)\right|\left(\frac{\beta_{4}}{2}|x|^{2 \alpha}+\frac{\beta_{3} k_{2}^{Q_{1}}+k \eta_{1}}{2}|x|^{\alpha}|\chi|^{\alpha}+\frac{k \eta_{2}}{2}|\chi|^{2 \alpha}\right)+\left|\xi_{2}(t)\right| \frac{k \beta_{5}}{2}|\chi|^{2 \alpha} .
\end{aligned}
$$

Hence, we obtain

$$
\begin{aligned}
& \left.\frac{d^{\alpha} \Xi}{d t^{\alpha}}\right|_{(6.3)} \leq-\beta_{\min }(M(\epsilon))\left(|x|^{2 \alpha}+|\chi|^{2 \alpha}\right)+\left|\xi_{1}(t)\right| \\
& \times\left(\frac{\beta_{4}}{2}|x|^{2 \alpha}+\frac{\beta_{3} k_{2}^{Q_{1}}+k \eta_{1}}{2}|x|^{\alpha}|\chi|^{\alpha}+\frac{k \eta_{2}}{2}|\chi|^{2 \alpha}\right)+\left|\xi_{2}(t)\right| \frac{k \beta_{5}}{2}|\chi|^{2 \alpha},
\end{aligned}
$$

where $\beta_{\min }(M(\epsilon))$ denotes the minimal eigenvalue of the following matrix

$$
M(\epsilon)=\left[\begin{array}{cc}
\frac{\beta_{1}}{2} & -\frac{\beta_{3} k_{2}^{F}+k \theta_{2}}{4} \\
-\frac{\beta_{3} k_{2}^{F}+k \theta_{2}}{4} & \frac{k}{2}\left(\frac{\beta_{3}}{\epsilon}-\gamma\right)
\end{array}\right] .
$$

From (7.6) we have

$$
\begin{aligned}
& \left.\frac{d^{\alpha} \Xi}{d t^{\alpha}}\right|_{(6.3)} \leq-\beta_{\min }(M(\epsilon))\left(|x|^{2 \alpha}+|\chi|^{2 \alpha}\right)+\left|\xi_{1}(t)\right| \\
& \times\left(\frac{\beta_{4}}{2}|x|^{2 \alpha}+\frac{\beta_{3} k_{2}^{Q_{1}}+k \eta_{1}}{2} \frac{\left(|x|^{2 \alpha}+|\chi|^{2 \alpha}\right)}{2}+\frac{k \eta_{2}}{2}|\chi|^{2 \alpha}\right)+\left|\xi_{2}(t)\right| \frac{k \beta_{5}}{2}|\chi|^{2 \alpha} .
\end{aligned}
$$

Let $\delta=\max \left\{\frac{\beta_{4}}{2}+\frac{\beta_{3} k_{2}^{Q_{1}}+k \eta_{1}}{2}, \frac{k \eta_{2}}{2}+\frac{\beta_{3} k_{2}^{Q_{1}}+k \eta_{1}}{2}\right\}$. Then

$$
\begin{aligned}
\left.\frac{d^{\alpha} \Xi}{d t^{\alpha}}\right|_{(6.3)} \leq & -\beta_{\min }(M(\epsilon))\left(|x|^{2 \alpha}+|\chi|^{2 \alpha}\right)+\left|\xi_{1}(t)\right| \delta\left(|x|^{2 \alpha}+|\chi|^{2 \alpha}\right) \\
& +\left|\xi_{2}(t)\right| \frac{k \beta_{5}}{2}|\chi|^{2 \alpha} .
\end{aligned}
$$

By noticing (7.5) we find

$$
\left.\frac{d^{\alpha} \Xi}{d t^{\alpha}}\right|_{(6.3)} \leq \frac{-2 \beta_{\min }(M(\epsilon))}{\beta_{h}} \Xi+\left|\xi_{1}(t)\right| \frac{2 \delta}{k \beta_{l}} \Xi+\left|\xi_{2}(t)\right| \frac{\beta_{5}}{\theta_{l}} \Xi .
$$

Employing Lemma 1, we get the estimate

$$
\begin{aligned}
\Xi(x(t), \chi(t)) \leq & \frac{1}{2} \Xi(x(0), \chi(0)) E_{\alpha}\left(\frac{-2 \beta_{\min }(M(\epsilon))}{\beta_{h}} t^{\alpha}\right) \\
& \times\left[E_{\alpha}\left(2 \int_{0}^{t} \frac{2 \delta}{k \theta_{l}}\left|\xi_{1}(s)\right|(d s)^{\alpha}\right)+E_{\alpha}\left(2 \int_{0}^{t} \frac{\beta_{5}}{\theta_{l}}\left|\xi_{1}(s)\right|(d s)^{\alpha}\right)\right] .
\end{aligned}
$$

Hence, using (6.4) we get

$$
\begin{aligned}
\mathbf{E}[\Xi & (x(t), \chi(t))] \leq \frac{1}{2} \Xi(x(0), \chi(0)) E_{\alpha}\left(\frac{-2 \lambda_{\min }(\epsilon)}{\beta_{h}} t^{\alpha}\right) \\
& \times\left[E_{\alpha}\left(\frac{4 \delta}{k \theta_{l}} k_{1} t^{\alpha}\right)+E_{\alpha}\left(\frac{2 \beta_{5}}{\theta_{l}} k_{2} t^{\alpha}\right)\right] .
\end{aligned}
$$


Finally

$$
\begin{aligned}
\mathbf{E}[\Xi( & x(t), \chi(t))] \leq \Xi(x(0), \chi(0)) E_{\alpha}\left(\frac{-2 \lambda_{\min }(\epsilon)}{\beta_{h}} t^{\alpha}\right) \\
& \times E_{\alpha}\left(\max \left\{\frac{4 \delta}{k \theta_{l}} k_{1}, \frac{2 \beta_{5}}{\theta_{l}} k_{2}\right\} t^{\alpha}\right) .
\end{aligned}
$$

But, the above relation is equivalent to

$$
\begin{aligned}
\mathbf{E}[\Xi(x(t), \chi(t))] \leq & \Xi(x(0), \chi(0)) E_{\alpha}\left(-\min \left\{\frac{2 \beta_{\min }(M(\epsilon))}{\beta_{h}}-\frac{4 \delta}{k \theta_{l}} k_{1},\right.\right. \\
& \left.\left.\frac{2 \beta_{\min }(M(\epsilon))}{\beta_{h}}-\frac{2 \beta_{5}}{\theta_{l}} k_{2}\right\} t^{\alpha}\right) .
\end{aligned}
$$

Using Lemma 2 and (6.4) we conclude that the singularly perturbed system (6.3) is Mittag-Leffler $\alpha$-mean square string stable for sufficiently small $\epsilon$.

Theorem 6. Assume that Assumptions 1-5 hold and additionally, the following assumptions is fulfilled:

$$
k_{0}=\min \left\{\frac{\beta_{1}}{\beta_{h}}-\frac{4 \delta}{k \theta_{l}} k_{1}, \frac{\beta_{1}}{\beta_{h}}-\frac{2 \beta_{5}}{\theta_{l}} k_{2}\right\}>\sum_{j=2}^{r} \frac{\beta_{1 j}}{\beta_{l}}+k \sum_{j=2}^{r} \frac{\gamma_{j}}{\beta_{l}} \geq 0,
$$

where

$$
k=\min \left\{\frac{\beta_{h}}{\theta_{h}}, \frac{\beta_{l}}{\theta_{l}}\right\}, \quad \delta=\max \left\{\frac{\beta_{4}}{2}+\frac{\beta_{3} k_{2}^{Q_{1}}+k \eta_{1}}{4}, \frac{k \eta_{2}}{2}+\frac{\beta_{3} k_{2}^{Q_{1}}+k \eta_{1}}{4}\right\} .
$$

Then, there exists a positive constant $\bar{\epsilon}$ such that, for each $\epsilon \in(0, \bar{\epsilon})$, the system (6.2) is Mittag-Leffler $\alpha$-mean square string stable.

Proof. We calculate $\frac{d^{\alpha} U_{i}}{d t^{\alpha}}$ for system (6.2)

$$
\begin{aligned}
& \left.\frac{d^{\alpha} U_{i}}{d t^{\alpha}}\right|_{(6.2)}=\frac{\partial^{\alpha} U_{i}}{\partial x^{i^{\alpha}}}\left(F\left(x^{i}, \chi^{i}+\zeta\left(x^{i}\right), x^{i-1}, \ldots, x^{i-r+1}\right)+Q_{1}\left(x^{i}, \chi^{i}+\zeta\left(x^{i}\right)\right) \xi_{1}^{i}\right) \\
& =\frac{\partial^{\alpha} U_{i}}{\partial x^{i^{\alpha}}}\left(F\left(x^{i}, \chi^{i}+\zeta\left(x^{i}\right), x^{i-1}, \ldots, x^{i-r+1}\right)+\frac{\partial^{\alpha} U_{i}}{\partial x^{i^{\alpha}}} Q_{1}\left(x^{i}, \zeta\left(x^{i}\right)\right) \xi_{1}^{i}\right. \\
& +\frac{\partial^{\alpha} U_{i}}{\partial x^{i^{\alpha}}}\left(F\left(x^{i}, \chi^{i}+\zeta\left(x^{i}\right), x^{i-1}, \ldots, x^{i-r+1}\right)-F\left(x^{i}, \zeta\left(x^{i}\right), x^{i-1}, \ldots, x^{i-r+1}\right)\right) \\
& +\frac{\partial^{\alpha} U_{i}}{\partial x^{i^{\alpha}}}\left(Q_{1}\left(x^{i}, \chi^{i}+\zeta\left(x^{i}\right)\right)-Q_{1}\left(x^{i}, \zeta\left(x^{i}\right)\right)\right) \xi_{1}^{i} .
\end{aligned}
$$

From Assumptions 2 and 4 we obtain

$$
\begin{aligned}
\left.\frac{d^{\alpha} U_{i}}{d t^{\alpha}}\right|_{(6.2)} \leq & -\beta_{1}\left|x^{i}\right|^{2 \alpha}+\beta_{3} k_{2}^{F}\left|x^{i}\right|^{\alpha}\left|\chi^{i}\right|^{\alpha}+\left|\xi_{1}^{i}\right|\left(\beta_{4}\left|x^{i}\right|^{2 \alpha}+\beta_{3} k_{2}^{Q_{1}}\left|x^{i}\right|^{\alpha}\left|\chi^{i}\right|^{\alpha}\right) \\
& +\sum_{j=2}^{r} \beta_{1 j}\left|x^{i-j+1}\right|^{2 \alpha} .
\end{aligned}
$$


Applying Assumptions 3 and 4 we obtain

$$
\begin{aligned}
\left.\frac{d^{\alpha} V_{i}}{d t^{\alpha}}\right|_{(6.2)} \leq & \theta_{2}\left|x^{i}\right|^{\alpha}\left|\chi^{i}\right|^{\alpha}+\gamma\left|\chi^{i}\right|^{2 \alpha}+\sum_{j=2}^{r} \gamma_{j}\left|x^{i-j+1}\right|^{2 \alpha}-\frac{\beta_{2}}{\epsilon}\left|\chi^{i}\right|^{2 \alpha} \\
& +\left|\xi_{1}^{i}\right|\left(\eta_{1}\left|x^{i}\right|^{\alpha}\left|\chi^{i}\right|^{\alpha}+\eta_{2}\left|\chi^{i}\right|^{2 \alpha}\right)+\left|\xi_{2}^{i}\right| \beta_{5}\left|\chi^{i}\right|^{2 \alpha} .
\end{aligned}
$$

Consider the function introduced by:

$$
\Xi_{i}=\Xi\left(x^{i}, \chi^{i}\right)=\frac{1}{2}\left[U\left(x^{i}\right)+k V\left(x^{i}, \chi^{i}\right)\right],
$$

where

$$
k=\min \left\{\beta_{h} / \theta_{h}, \beta_{l} / \theta_{l}\right\} .
$$

From Assumptions 2 and 3 we have

$$
\frac{\beta_{l}\left|x^{i}\right|^{2 \alpha}+k \theta_{l}\left|\chi^{i}\right|^{2 \alpha}}{2} \leq \Xi_{i} \leq \frac{\beta_{h}\left|x^{i}\right|^{2 \alpha}+k \theta_{h}\left|\chi^{i}\right|^{2 \alpha}}{2} .
$$

Using (7.9) and (7.10) we have

$$
\left|x^{i}\right|^{2 \alpha}+\left|\chi^{i}\right|^{2 \alpha} \geq \frac{2 \Xi_{i}}{\beta_{h}}, \quad\left|x^{i}\right|^{2 \alpha}+\left|\chi^{i}\right|^{2 \alpha} \leq \frac{2 \Xi_{i}}{k \theta_{l}} .
$$

We can calculate $\frac{d^{\alpha} \Xi_{i}}{d t^{\alpha}}$ for system (6.2) as follows

$$
\left.\frac{d^{\alpha} \Xi_{i}}{d t^{\alpha}}\right|_{(6.2)}=\frac{1}{2} \frac{d^{\alpha} U_{i}}{d t^{\alpha}}+\frac{k}{2} \frac{d^{\alpha} V_{i}}{d t^{\alpha}} .
$$

From relations (7.7) and (7.8) we have

$$
\begin{aligned}
& \left.\frac{d^{\alpha} \Xi_{i}}{d t^{\alpha}}\right|_{(6.2)} \leq-\left(\frac{\beta_{1}}{2}\left|x^{i}\right|^{2 \alpha}-\frac{\beta_{3} k_{2}^{F}+k \theta_{2}}{2}\left|x^{i}\right|^{\alpha}\left|\chi^{i}\right|^{\alpha}+\frac{k}{2}\left(\frac{\beta_{3}}{\epsilon}-\gamma\right)\left|\chi^{i}\right|^{2 \alpha}\right) \\
& +\sum_{j=2}^{r} \frac{\beta_{1 j}+k \gamma_{j}}{2}\left|x^{i-j+1}\right|^{2 \alpha}+\left|\xi_{1}^{i}\right| \\
& \quad \times\left(\frac{\beta_{4}}{2}\left|x^{i}\right|^{2 \alpha}+\frac{\beta_{3} k_{2}^{Q_{1}}+k \eta_{1}}{2}\left|x^{i}\right|^{\alpha}\left|\chi^{i}\right|^{\alpha}+\frac{k \eta_{2}}{2}\left|\chi^{i}\right|^{2 \alpha}\right)+\left|\xi_{2}^{i}\right| \frac{k \beta_{5}}{2}\left|\chi^{i}\right|^{2 \alpha} .
\end{aligned}
$$

Consequently, one will set

$$
\begin{aligned}
& \left.\frac{d^{\alpha} \Xi_{i}}{d t^{\alpha}}\right|_{(6.2)} \leq-\beta_{\min }(M(\epsilon))\left(\left|x^{i}\right|^{2 \alpha}+\left|\chi^{i}\right|^{2 \alpha}\right)+\sum_{j=2}^{r} \frac{\beta_{1 j}+k \gamma_{j}}{2}\left|x^{i-j+1}\right|^{2 \alpha} \\
& +\left|\xi_{1}^{i}\right|\left(\frac{\beta_{4}}{2}\left|x^{i}\right|^{2 \alpha}+\frac{\beta_{3} k_{2}^{Q_{1}}+k \eta_{1}}{2}\left|x^{i}\right|^{\alpha}\left|\chi^{i}\right|^{\alpha}+\frac{k \eta_{2}}{2}\left|\chi^{i}\right|^{2 \alpha}\right)+\left|\xi_{2}^{i}\right| \frac{k \beta_{5}}{2}\left|\chi^{i}\right|^{2 \alpha},
\end{aligned}
$$

where $\beta_{\min }(M(\epsilon))$ denotes the minimal eigenvalue of the following matrix

$$
M(\epsilon)=\left[\begin{array}{cc}
\frac{\beta_{1}}{2} & -\frac{\beta_{3} k_{2}^{F}+k \theta_{2}}{4} \\
-\frac{\beta_{3} k_{2}^{F}+k \theta_{2}}{4} & \frac{k}{2}\left(\frac{\beta_{3}}{\epsilon}-\gamma\right)
\end{array}\right],
$$


and hence

$$
\begin{aligned}
& \left.\frac{d^{\alpha} \Xi_{i}}{d t^{\alpha}}\right|_{(6.2)} \leq-\beta_{\min }(M(\epsilon))\left(\left|x^{i}\right|^{2 \alpha}+\left|\chi^{i}\right|^{2 \alpha}\right)+\sum_{j=2}^{r} \frac{\beta_{1 j}+k \gamma_{j}}{2}\left|x^{i-j+1}\right|^{2 \alpha} \\
& +\left|\xi_{1}^{i}\right|\left(\frac{\beta_{4}}{2}\left|x^{i}\right|^{2 \alpha}+\frac{\beta_{3} k_{2}^{Q_{1}}+k \eta_{1}}{2} \frac{\left(\left|x^{i}\right|^{2 \alpha}+\left|\chi^{i}\right|^{2 \alpha}\right)}{2}+\frac{k \eta_{2}}{2}\left|\chi^{i}\right|^{2 \alpha}\right)+\left|\xi_{2}^{i}\right| \frac{k \beta_{5}}{2}\left|\chi^{i}\right|^{2 \alpha} .
\end{aligned}
$$

Let $\delta=\max \left\{\frac{\beta_{4}}{2}+\frac{\beta_{3} k_{2}^{Q_{1}}+k \eta_{1}}{2}, \frac{k \eta_{2}}{2}+\frac{\beta_{3} k_{2}^{Q_{1}}+k \eta_{1}}{2}\right\}$. Then

$$
\begin{aligned}
\left.\frac{d^{\alpha} \Xi_{i}}{d t^{\alpha}}\right|_{(6.2)} \leq & -\beta_{\min }(M(\epsilon))\left(\left|x^{i}\right|^{2 \alpha}+\left|\chi^{i}\right|^{2 \alpha}\right)+\sum_{j=2}^{r} \frac{\beta_{1 j}+k \gamma_{j}}{2}\left|x^{i-j+1}\right|^{2 \alpha} \\
& +\left|\xi_{1}^{i}\right| \delta\left(\left|x^{i}\right|^{2 \alpha}+\left|\chi^{i}\right|^{2 \alpha}\right)+\left|\xi_{2}^{i}\right| \frac{k \beta_{5}}{2}\left|\chi^{i}\right|^{2 \alpha} .
\end{aligned}
$$

By noticing (7.11) we obtain

$$
\left.\frac{d^{\alpha} \Xi_{i}}{d t^{\alpha}}\right|_{(6.2)} \leq \frac{-2 \beta_{\min }(M(\epsilon))}{\beta_{h}} \Xi_{i}+\sum_{j=2}^{r} \frac{\beta_{1 j}+k \gamma_{j}}{\beta_{l}} \Xi_{i-j+1}+\left|\xi_{1}^{i}\right| \frac{2 \delta}{k \beta_{l}} \Xi_{i}+\left|\xi_{2}^{i}\right| \frac{\beta_{5}}{\theta_{l}} \Xi_{i} .
$$

Using Lemmas 2 and 5 we conclude that the singularly perturbed system (6.2) is Mittag-Leffler $\alpha$-mean square string stable for sufficiently small $\epsilon$.

\section{Conclusions}

The present study aimed to propose a new type of string stability based on Mittag-Leffler function called Mittag-Leffler string stability. The problem of Mittag-Leffler string stability for singularly perturbed nonlinear stochastic systems of fractional order has been considered. The sufficient conditions to guarantee the Mittag-Leffler string stability for interconnected stochastic systems were presented and their robustness to small singular perturbations was also demonstrated.

\section{References}

[1] T. Abdeljawad, E. Gundogdu and D. Baleanu. On the Mittag-Leffler stability of $q$-fractional nonlinear dynamical systems. Proc. Romanian Acad., 12:309-314, 2011.

[2] D. Baleanu, K. Diethelm, E. Scalas and J.J. Trujillo. Fractional calculus models and numerical methods. World Scientific, 2012. https://doi.org/10.1142/8180.

[3] H. Delavari, D. Baleanu and J. Sadati. Stability analysis of Caputo fractionalorder nonlinear systems revisited. Nonlinear Dynam., 67:2433-2439, 2012. https://doi.org/10.1007/s11071-011-0157-5.

[4] J.L. Doob. Stochastic processes. Wiley Chapman and Hall, New York, 1953.

[5] E.B. Dynkin. Markov processes. In Fizmatgiz, Moscow, 1963, English transl. Die Grundlehren der Math., p. 122, Wissenschaften, Bande 121, 1965. Academic Press, New York. 
[6] M. El-Ansary. Stochastic feedback design for a class of nonlinear singularly perturbed systems. Int. J. Syst. Sci., 22:2013-2023, 1991. https://doi.org/10.1080/00207729108910767.

[7] M. El-Ansary and H.K. Khalil. On the interplay of singular perturbations and wide-band stochastic fluctuations. SIAM J. Control Optim., 24:83-94, 1986. https://doi.org/10.1137/0324004.

[8] S.A. El-Shehawy. On properties of fractional probability measure. Int. Math. Forum, 11:1175-1184, 2016. https://doi.org/10.12988/imf.2016.610132.

[9] I.I. Gikhman and A.V. Skorokhod. Introduction to the theory of random processes. In Nauka, Moscow 1965, English transl. Saunders, Philadelphia, 1969.

[10] R. Gorenflo, A. Kilbas, F. Mainardi and S. Rogosin. Mittag-Leffler functions, related topics and applications. Springer-Verlag, Berlin-Heidelberg, 2014.

[11] H.J. Haubold, A.M. Mathai and R.K. Saxena. Mittag-Leffler functions and their applications. arXiv:0909.0230v2 [math.CA], 4:1-51, 2009.

[12] R.Z. Khasminski. Stochastic stability of differential equations. Groningen, The Netherlands: Sijthoff and Noordhoff, 1980. https://doi.org/10.1007/978-94-0099121-7.

[13] A. Kilbas, H.M. Srivastava and J.J. Trujillo. Theory and applications of fractional differential equations. Elsevier, Amsterdam, 2006.

[14] K.M. Kolwankar and A.D. Gangal. Fractional differentiability of nowhere differentiable functions and dimensions. Chaos, 6(4):505-513, 1996. https://doi.org/10.1063/1.166197.

[15] Y. Li, Y.Q. Chen and I. Podlubny. Stability of fractional-order nonlinear dynamic systems: Lyapunov direct method and generalized Mittag-Leffler stability. Comput. Math. Appl., 59:1810-1821, 2010. https://doi.org/10.1016/j.camwa.2009.08.019.

[16] M. Liao, X.J. Yang and Q. Yan. A new viewpoint to Fourier analysis in fractal space. Springer, New York, 2013. https://doi.org/10.1007/978-1-4614-6393$1 \_26$.

[17] M. Loeve. Probability theory. Foundations. Random sequences. Van Nostrand, Princeton, 1960.

[18] G. Mittag-Leffler. Sur la nouvelle fonction e(x). C. R. Acad. Sci. Paris, 137:554$558,1903$.

[19] H. Mostafaei and P.A. Ghotbi. Fractional probability measure and its properties. J. Sci. Islamic Rep. Iran, 21:259-264, 2010.

[20] V.S. Pugachev. The theory of random functions and its application to control problems. In Fizmatgiz, Moscow, 1960, volume 5 of English transl. Internat. Series of Monographs on Automation and Automatic Control. Pergamon Press, Oxford, Addison-Wesley, 1965.

[21] L. Rybarska-Rusinek. String stability of singularly perturbed stochastic systems. Ann. Math. Sil., 16:43-55, 2003.

[22] M. Zeki Sarikaya and H. Budak. Generalized Ostrowski type inequalities for local fractional integrals. P. Am. Math. Soc., 145(4):1527-1538, 2015.

[23] K. Sayevand and K. Pichaghchi. Successive approximation: A survey on stable manifold of fractional differential systems. Fract. Calculus Appl. Anal., 18:621641, 2015. https://doi.org/10.1515/fca-2015-0038. 
[24] M.V. Simkin and V.P. Roychowdhury. Stochastic modeling of a serial killer. J. Theor. Biol., 355:111-116, 2014. https://doi.org/10.1016/j.jtbi.2014.03.039.

[25] L. Socha. Stochastic stability of interconnected string systems. Chaos, Soliton. Fract., 19:949-955, 2004. https://doi.org/10.1016/S0960-0779(03)00266-2.

[26] D. Swaroop and J.K. Hedrick. String stability of interconnected systems. IEEE Trans. Autom. Control, 41:349-356, 1996. https://doi.org/10.1109/9.486636.

[27] B.J. West. A fractional probability calculus view of Allometry. Systems, 2:89118, 2014. https://doi.org/10.3390/systems2020089.

[28] X.J. Yang. Local fractional functional analysis and its applications. Asian Academic Publisher, Hong Kong, 2011.

[29] X.J. Yang. Local fractional integral transforms. Prog. Nonlinear Scie., 4:1-225, 2011.

[30] X.J. Yang. Advanced local fractional calculus and its applications. World Science Publisher, New York, 2012.

[31] X.J. Yang, D. Baleanu and H.M. Srivastava. Local fractional integral transforms and their applications. Academic Press, New York, 2015.

[32] X.J. Yang, F. Gao, J.A. Tenreiro Machado and D. Baleanu. Exact travelling wave solutions for local fractional partial differential equations in mathematical physics. Math. Methods Eng., 24:175-191, 2019. https://doi.org/10.1007/978-3319-90972-1_12.

[33] X.J. Yang, F. Gao and H.M. Srivastava. Exact travelling wave solutions for the local fractional two-dimensional Burgers-type equations. Compu. Math. Appl., 73(2):203-210, 2017. https://doi.org/10.1016/j.camwa.2016.11.012.

[34] X.J. Yang, F. Gao and H.M. Srivastava. A new computational approach for solving nonlinear local fractional PDEs. J. Comput. Appl. Math., 339:285-296, 2018. https://doi.org/10.1016/j.cam.2017.10.007. 